\title{
Research Article \\ Complex Transforms for Systems of Fractional Differential Equations
}

\author{
Rabha W. Ibrahim \\ Institute of Mathematical Sciences, University of Malaya, 50603 Kuala Lumpur, Malaysia \\ Correspondence should be addressed to Rabha W. Ibrahim, rabhaibrahim@yahoo.com
}

Received 8 August 2012; Revised 11 October 2012; Accepted 11 October 2012

Academic Editor: Elena Litsyn

Copyright ( 2012 Rabha W. Ibrahim. This is an open access article distributed under the Creative Commons Attribution License, which permits unrestricted use, distribution, and reproduction in any medium, provided the original work is properly cited.

We provide a complex transform that maps the complex fractional differential equation into a system of fractional differential equations. The homogeneous and nonhomogeneous cases for equivalence equations are discussed and also nonequivalence equations are studied. Moreover, the existence and uniqueness of solutions are established and applications are illustrated.

\section{Introduction}

Transform is a significant method to solve mathematical problems. Various practical transforms for solving various problems were materialized in open literature, such as the Laplace transform [1], the Fourier transform [2], the traveling wave transform [3], the Bäcklund transformation [4], the integral transform [5], the fractional integral transforms [6], the fractional complex transform [7, 8], and Mellin transform [9].

Fractional models have been studied by many researchers to sufficiently describe the operation of variety of computational, physical, and biological processes and systems. Accordingly, considerable attention has been paid to the solution of fractional differential equations, integral equations, and fractional partial differential equations of physical phenomena. Most of these fractional differential equations have analytic solutions, approximation, and numerical techniques [10-12]. Numerical and analytical methods have included finite difference method such as Adomian decomposition method, variational iteration method, homotopy perturbation method, and homotopy analysis method [13-16].

One of the most frequently used tools in the theory of fractional calculus is furnished by the Riemann-Liouville operators. It possesses advantages of fast convergence, higher stability, and higher accuracy to derive different types of numerical algorithms. In this paper, we will deal with scalar linear time-fractional differential equations. The time is taken in 
sense of the Riemann-Liouville fractional operators. Also, This type of differential equations arises in many interesting applications. For example the fractional Fokker-Planck differential equation which can be derived from the master equation [17], the fractional diffusion equations [18-23], and the generalized Chapman-Kolmogorov equation [24].

Here, we shall use the complex transform to reduce this fractional differential equation to a system of fractional order (homogeneous and nonhomogeneous) keeping the equivalency properties. Furthermore, we shall study the existence and uniqueness of solutions for the linear and nonlinear cases of these systems. Applications are imposed.

\section{Fractional Calculus}

The idea of the fractional calculus (i.e., calculus of integrals and derivatives of any arbitrary real or complex order) was planted over 300 years ago. Abel in 1823 investigated the generalized tautochrone problem and for the first time applied fractional calculus techniques in a physical problem. Later Liouville applied fractional calculus to problems in potential theory. Since that time the fractional calculus has haggard the attention of many researchers in all area of sciences (see [25-27]).

This section concerns with some preliminaries and notations regarding the fractional calculus.

Definition 2.1. The fractional (arbitrary) order integral of the function $f$ of order $\alpha>0$ is defined by

$$
I_{a}^{\alpha} f(t)=\int_{a}^{t} \frac{(t-\tau)^{\alpha-1}}{\Gamma(\alpha)} f(\tau) d \tau
$$

When $=0$, we write $I_{a}^{\alpha} f(t)=f(t) * \phi_{\alpha}(t)$, where $(*)$ denoted the convolution product (see [16]), $\phi_{\alpha}(t)=t^{\alpha-1} / \Gamma(\alpha), t>0, \phi_{\alpha}(t)=0, t \leq 0$ and $\phi_{\alpha} \rightarrow \delta(t)$ as $\alpha \rightarrow 0$ where $\delta(t)$ is the delta function.

Definition 2.2. The fractional (arbitrary) order derivative of the function $f$ of order $0 \leq \alpha<1$ is defined by

$$
D_{a}^{\alpha} f(t)=\frac{d}{d t} \int_{a}^{t} \frac{(t-\tau)^{-\alpha}}{\Gamma(1-\alpha)} f(\tau) d \tau \frac{d}{d t} I_{a}^{1-\alpha} f(t)
$$

Remark 2.3. From Definitions 2.1 and 2.2, $a=0$, we have

$$
\begin{aligned}
D^{\alpha} t^{\mu} & =\frac{\Gamma(\mu+1)}{\Gamma(\mu-\alpha+1)} t^{\mu-\alpha}, \quad \mu>-1 ; 0<\alpha<1, \\
I^{\alpha} t^{\mu} & =\frac{\Gamma(\mu+1)}{\Gamma(\mu+\alpha+1)} t^{\mu+\alpha}, \quad \mu>-1 ; \alpha>0 .
\end{aligned}
$$


The Leibniz rule is

$$
D_{a}^{\alpha}[f(t) g(t)]=\sum_{k=0}^{\infty}\left(\begin{array}{l}
\alpha \\
k
\end{array}\right) D_{a}^{\alpha-k} f(t) D_{a}^{k} g(t)=\sum_{k=0}^{\infty}\left(\begin{array}{l}
\alpha \\
k
\end{array}\right) D_{a}^{\alpha-k} g(t) D_{a}^{k} f(t)
$$

where

$$
\left(\begin{array}{l}
\alpha \\
k
\end{array}\right)=\frac{\Gamma(\alpha+1)}{\Gamma(k+1) \Gamma(\alpha+1-k)}
$$

In this paper we deal with the following fractional differential equation:

$$
D^{\alpha} u(t, z)=a(t, z) u_{z z}+b(t, z) u_{z}+c(t, z) u
$$

where $a \neq 0, b, c, u$ are complex valued functions, analytic in the domain $\Phi:=J \times U$; $J=$ $[0, T], T \in(0, \infty)$, and $U:=\{z \in \mathbb{C},|z| \leq 1\}$.

Definition 2.4. The Caputo fractional derivative of order $\mu>0$ is defined, for a smooth function $f(t)$, by

$$
{ }^{c} D^{\mu} f(t):=\frac{1}{\Gamma(n-\mu)} \int_{0}^{t} \frac{f^{(n)}(\zeta)}{(t-\zeta)^{\mu-n+1}} d \zeta
$$

where $n=[\mu]+1$ (the notation $[\mu]$ stands for the largest integer not greater than $\mu$ ).

Note that there is a relationship between Riemann-Liouville differential operator and the Caputo operator

$$
D_{a}^{\mu} f(t)=\frac{1}{\Gamma(1-\mu)} \frac{f(a)}{(t-a)^{\mu}}+{ }^{c} D_{a}^{\mu} f(t)
$$

and they are equivalent in a physical problem (i.e., a problem which specifies the initial conditions) [28].

Equation (2.6) involves well known time fractional diffusion equations. Several researchers have studied fractional dynamic equations generalizing the diffusion or wave equations in terms of R-L or Caputo time fractional derivatives, and their fundamental solutions have been represented in terms of the Mittag-Leffler (M-L) functions and their generalizations. The mathematical study of fractional diffusion equations began with the work of Kochuber [29, 30]. Later this study followed by the work of Metzler and Klafter [31] and Zaslavsky [32]. Recently, Mainardi et al. obtained the time fractional diffusion equation from the standard diffusion equation [33, 34]. By using the Fourier-Laplace transform method, it was shown in [35] that the fundamental solution of the fractional diffusion equation with a generalized Riemann-Liouville time fractional derivative defined in the infinite domain can be expressed via Foxs $H$-function, when $a(t, z)=K$, where $K$ is a constant. Moreover, by employing the concept of majorant functions, it was proved in [36] the existence and uniqueness of holomorphic solutions to nonlinear fractional diffusion problems. Finally, by 
applying the fractional variational iteration method, it was provided in [37] the numerical solutions of time-fractional reaction-diffusion equation with modified Riemann-Liouville derivative.

\section{Complex Transforms}

In this section, we shall illustrate two types of complex transform. The first type is equivalence transforms for the homogeneous and nonhomogeneous equations. The second is non equivalence transform.

\subsection{Equivalence Transforms}

Assume the complex transform

$$
u(t, z)=\sigma(z) \bar{u}(t, z)
$$

where $\sigma \neq 0$ is a complex valued function of complex variable $z \in U$; therefor, we have

$$
\begin{array}{cc}
\sigma(z):=\sigma_{1}(z)+i \sigma_{2}(z), & u(t, z)=v(t, z)+i w(t, z), \\
a(t, z)=a_{1}(t, z)+i a_{2}(t, z), & b(t, z)=b_{1}(t, z)+i b_{2}(t, z), \\
c(t, z)=c_{1}(t, z)+i c_{2}(t, z), & \bar{u}(t, z)=\bar{v}(t, z)+i \bar{w}(t, z) .
\end{array}
$$

Substituting (3.1) in (2.6) yields

$$
D^{\alpha} \bar{u}(t, z)=\bar{a}(t, z) \bar{u}_{z z}+\bar{b}(t, z) \bar{u}_{z}+\bar{c}(t, z) \bar{u}
$$

where

$$
\bar{a}=a, \quad \bar{b}=b+2 a \frac{\sigma_{z}}{\sigma}, \quad \bar{c}=c+a \frac{\sigma_{z z}}{\sigma}+b \frac{\sigma_{z}}{\sigma} .
$$

Thus under the map (3.1), (2.6) remains homogeneous with new coefficients. Our aim is to translate (3.3) into a system of fractional order using the relations in (3.2). Again substituting (3.2) in (2.6) implies the following system:

$$
\begin{aligned}
& D^{\alpha} v=a_{1} v_{z z}-a_{2} w_{z z}+b_{1} v_{z}-b_{2} w_{z}+c_{1} v-c_{2} w \\
& D^{\alpha} w=a_{1} w_{z z}+a_{2} v_{z z}+b_{1} w_{z}+b_{2} v_{z}+c_{1} w+c_{2} v
\end{aligned}
$$

Since $u=v+i w=\left(\sigma_{1}(z)+i \sigma_{2}(z)\right)(\bar{v}(t, z)+i \bar{w}(t, z))=\sigma_{1} \bar{v}-\sigma_{2} \bar{w}+i\left(\sigma_{1} \bar{w}+\sigma_{2} \bar{v}\right)$, then we obtain the relations

$$
\begin{aligned}
v & =\sigma_{1} \bar{v}-\sigma_{2} \bar{w}, \\
w & =\sigma_{2} \bar{v}+\sigma_{1} \bar{w} .
\end{aligned}
$$


Consequently we have

$$
\begin{gathered}
v_{z}=\sigma_{1} \bar{v}_{z}+\sigma_{1 z} \bar{v}-\sigma_{2} \bar{w}_{z}-\sigma_{2 z} \bar{w} \\
v_{z z}=\sigma_{1} \bar{v}_{z z}+2 \sigma_{1 z} \bar{v}_{z}+\sigma_{1 z z} \bar{v}-\sigma_{2} \bar{w}_{z z}-2 \sigma_{2 z} \bar{w}_{z}-\sigma_{2 z z} \bar{w} \\
w_{z}=\sigma_{2} \bar{v}_{z}+\sigma_{2 z} \bar{v}+\sigma_{1} \bar{w}_{z}+\sigma_{1 z} \bar{w} \\
v_{z z}=\sigma_{2} \bar{v}_{z z}+2 \sigma_{2 z} \bar{v}_{z}+\sigma_{2 z z} \bar{v}+\sigma_{1} \bar{w}_{z z}+2 \sigma_{1 z} \bar{w}_{z}+\sigma_{1 z z} \bar{w} .
\end{gathered}
$$

By the linearity of the fractional differential operator $D^{\alpha}$, we impose that

$$
\begin{aligned}
D^{\alpha} v & =\sigma_{1} D^{\alpha} \bar{v}-\sigma_{2} D^{\alpha} \bar{w} \\
D^{\alpha} w & =\sigma_{2} D^{\alpha} \bar{v}+\sigma_{1} D^{\alpha} \bar{w} .
\end{aligned}
$$

By injecting (3.7) and (3.8) in (3.5) we receive the following system:

$$
\begin{aligned}
& D^{\alpha} \bar{v}=\bar{a}_{1} \bar{v}_{z z}-\bar{a}_{2} \bar{w}_{z z}+\bar{b}_{1} \bar{v}_{z}-\bar{b}_{2} \bar{w}_{z}+\bar{c}_{1} \bar{v}-\bar{c}_{2} \bar{w} \\
& D^{\alpha} \bar{w}=\bar{a}_{1} \bar{w}_{z z}+\bar{a}_{2} \bar{v}_{z z}+\bar{b}_{1} \bar{w}_{z}+\bar{b}_{2} \bar{v}_{z}+\bar{c}_{1} \bar{w}+\bar{c}_{2} \bar{v}
\end{aligned}
$$

where

$$
\begin{gathered}
\bar{a}_{1}=a_{1}, \quad \bar{a}_{2}=a_{2}, \\
\bar{b}_{1}=b_{1}+\frac{2 \sigma_{1 z}\left(a_{1} \sigma_{1}+a_{2} \sigma_{2}\right)+2 \sigma_{2 z}\left(a_{1} \sigma_{2}-a_{2} \sigma_{1}\right)}{\sigma_{1}^{2}+\sigma_{2}^{2}} \\
\bar{b}_{2}=b_{2}-\frac{2 \sigma_{1 z}\left(a_{1} \sigma_{2}-a_{2} \sigma_{1}\right)+2 \sigma_{2 z}\left(a_{2} \sigma_{2}-a_{1} \sigma_{1}\right)}{\sigma_{1}^{2}+\sigma_{2}^{2}}, \\
\bar{c}_{1}=c_{1}+\frac{\sigma_{1 z z}\left(a_{1} \sigma_{1}+a_{2} \sigma_{2}\right)+\sigma_{2 z z}\left(a_{1} \sigma_{2}-a_{2} \sigma_{1}\right)+\sigma_{1 z}\left(b_{1} \sigma_{1}+b_{2} \sigma_{2}\right)+\sigma_{2 z}\left(b_{1} \sigma_{2}-b_{2} \sigma_{1}\right)}{\sigma_{1}^{2}+\sigma_{2}^{2}}, \\
\bar{c}_{2}=c_{2}-\frac{\sigma_{1 z z}\left(a_{1} \sigma_{2}-a_{2} \sigma_{1}\right)+\sigma_{2 z z}\left(a_{1} \sigma_{1}-a_{2} \sigma_{2}\right)+\sigma_{1 z}\left(b_{1} \sigma_{2}-b_{2} \sigma_{1}\right)-\sigma_{2 z}\left(b_{1} \sigma_{1}+b_{2} \sigma_{2}\right)}{\sigma_{1}^{2}+\sigma_{2}^{2}}
\end{gathered}
$$

Hence we conclude the following result.

Theorem 3.1. Let $a \neq 0, b, c, u$ be complex valued functions, analytic in the domain $\Phi=J \times U$, and let $\sigma \neq 0$ be complex valued function in $U$. Then the fractional coupled system (3.5) is equivalent to the transformed fractional coupled system (3.9) if and only if (3.10) holds. 
Corollary 3.2. Let

$$
\begin{gathered}
a_{2}=0, \\
b_{2}=\frac{2 \sigma_{1 z} a_{1} \sigma_{2}-2 \sigma_{2 z} a_{1} \sigma_{1}}{\sigma_{1}^{2}+\sigma_{2}^{2}}, \\
c_{2}=\frac{\sigma_{1 z z} a_{1} \sigma_{2}+\sigma_{2 z z} a_{1} \sigma_{1}+\sigma_{1 z}\left(b_{1} \sigma_{2}-b_{2} \sigma_{1}\right)-\sigma_{2 z}\left(b_{1} \sigma_{1}+b_{2} \sigma_{2}\right)}{\sigma_{1}^{2}+\sigma_{2}^{2}} .
\end{gathered}
$$

Then the fractional coupled system (3.5) is equivalent to the transformed fractional uncoupled system

$$
\begin{aligned}
D^{\alpha} \bar{v} & =\bar{a}_{1} \bar{v}_{z z}+\bar{b}_{1} \bar{v}_{z}+\bar{c}_{1} \bar{v}, \\
D^{\alpha} \bar{w} & =\bar{a}_{1} \bar{w}_{z z}+\bar{b}_{1} \bar{w}_{z}+\bar{c}_{1} \bar{w},
\end{aligned}
$$

where $\bar{a}_{1}, \bar{b}_{1}$, and $\bar{c}_{1}$ are defined in (3.10).

Now we proceed to consider the nonhomogeneous equation

$$
D^{\alpha} u(t, z)=a(t, z) u_{z z}+b(t, z) u_{z}+c(t, z) u+f(t, z)
$$

where $a \neq 0, b, c, u$, and $f$ are complex valued functions, analytic in the domain $\Phi$ such that $f=$ $f_{1}+i f_{2}$.

Assume the complex transform

$$
u(t, z)=\rho(t, z) \bar{u}(t, z)
$$

where $\rho \neq 0$ is a complex valued function of complex variable $z \in U$, with

$$
\rho(t, z):=\rho_{1}(t, z)+i \rho_{2}(t, z)
$$

Substituting (3.14) in (3.13) and using the Leibniz rule imply

$$
D^{\alpha} \bar{u}(t, z)=\bar{a}(t, z) \bar{u}_{z z}+\bar{b}(t, z) \bar{u}_{z}+\bar{c}(t, z) \bar{u}+\bar{f}(t, z),
$$

where

$$
\bar{a}=a, \quad \bar{b}=b+2 a \frac{\rho_{z}}{\rho}, \quad \bar{c}=c+a \frac{\rho_{z z}}{\rho}+b \frac{\rho_{z}}{\rho}, \quad \bar{f}=\frac{f}{\rho}-\frac{\alpha \rho_{t}}{\rho} I^{1-\alpha} \bar{u} .
$$

Obviously, (3.16) remains nonhomogeneous with new coefficients. Our goal is to reduce (3.16) to a system of fractional order using the relations (3.2) and (3.15). A calculation yields the following system:

$$
\begin{aligned}
D^{\alpha} v & =a_{1} v_{z z}-a_{2} w_{z z}+b_{1} v_{z}-b_{2} w_{z}+c_{1} v-c_{2} w+f_{1} \\
D^{\alpha} w & =a_{1} w_{z z}+a_{2} v_{z z}+b_{1} w_{z}+b_{2} v_{z}+c_{1} w+c_{2} v+f_{2} .
\end{aligned}
$$


Since

$$
\begin{gathered}
v=\rho_{1} \bar{v}-\rho_{2} \bar{w}, \\
w=\rho_{2} \bar{v}+\rho_{1} \bar{w},
\end{gathered}
$$

then by taking the fractional derivative of the variables $v$ and $w$ in (3.19), we obtain

$$
\begin{aligned}
& D^{\alpha} v=\rho_{1} D^{\alpha} \bar{v}-\rho_{2} D^{\alpha} \bar{w}+h_{1} \\
& D^{\alpha} w=\rho_{2} D^{\alpha} \bar{v}+\rho_{1} D^{\alpha} \bar{w}+h_{2}
\end{aligned}
$$

where

$$
\begin{aligned}
& h_{1}=\rho_{1 t} I^{1-\alpha} \bar{v}-\rho_{2 t} I^{1-\alpha} \bar{w}, \\
& h_{2}=\rho_{2 t} I^{1-\alpha} \bar{v}+\rho_{1 t} I^{1-\alpha} \bar{w} .
\end{aligned}
$$

Consequently, we impose the following system:

$$
\begin{aligned}
& D^{\alpha} \bar{v}=\bar{a}_{1} \bar{v}_{z z}-\bar{a}_{2} \bar{w}_{z z}+\bar{b}_{1} \bar{v}_{z}-\bar{b}_{2} \bar{w}_{z}+\bar{c}_{1} \bar{v}-\bar{c}_{2} \bar{w}+\bar{f}_{1}, \\
& D^{\alpha} \bar{w}=\bar{a}_{1} \bar{w}_{z z}+\bar{a}_{2} \bar{v}_{z z}+\bar{b}_{1} \bar{w}_{z}+\bar{b}_{2} \bar{v}_{z}+\bar{c}_{1} \bar{w}+\bar{c}_{2} \bar{v}+\bar{f}_{2}
\end{aligned}
$$

where

$$
\begin{gathered}
\bar{a}_{1}=a_{1}, \quad \bar{a}_{2}=a_{2}, \\
\bar{b}_{1}=b_{1}+\frac{2 \rho_{1 z}\left(a_{1} \rho_{1}+a_{2} \rho_{2}\right)+2 \rho_{2 z}\left(a_{1} \rho_{2}-a_{2} \rho_{1}\right)}{\rho_{1}^{2}+\rho_{2}^{2}}, \\
\bar{b}_{2}=b_{2}-\frac{2 \rho_{1 z}\left(a_{1} \rho_{2}-a_{2} \rho_{1}\right)+2 \rho_{2 z}\left(a_{2} \rho_{2}-a_{1} \rho_{1}\right)}{\rho_{1}^{2}+\rho_{2}^{2}}, \\
\bar{c}_{1}=c_{1}+\frac{\rho_{1 z z}\left(a_{1} \rho_{1}+a_{2} \rho_{2}\right)+\rho_{2 z z}\left(a_{1} \rho_{2}-a_{2} \rho_{1}\right)+\rho_{1 z}\left(b_{1} \rho_{1}+b_{2} \rho_{2}\right)+\rho_{2 z}\left(b_{1} \rho_{2}-b_{2} \rho_{1}\right)}{\rho_{1}^{2}+\rho_{2}^{2}}, \\
\bar{c}_{2}=c_{2}-\frac{\rho_{1 z z}\left(a_{1} \rho_{2}-a_{2} \rho_{1}\right)+\rho_{2 z z}\left(a_{1} \rho_{1}-a_{2} \rho_{2}\right)+\rho_{1 z}\left(b_{1} \rho_{2}-b_{2} \rho_{1}\right)-\rho_{2 z}\left(b_{1} \rho_{1}+b_{2} \rho_{2}\right)}{\rho_{1}^{2}+\rho_{2}^{2}}, \\
\bar{f}_{1}=\frac{\rho_{1}\left(f_{1}-h_{1}\right)+\rho_{2}\left(f_{2}-h_{2}\right)}{\rho_{1}^{2}+\rho_{2}^{2}}, \\
\bar{f}_{2}=\frac{\rho_{2}\left(f_{1}-h_{1}\right)+\rho_{1}\left(f_{2}-h_{2}\right)}{\rho_{1}^{2}+\rho_{2}^{2}} .
\end{gathered}
$$

Hence we conclude the following result. 
Theorem 3.3. Let $a \neq 0, b, c, u, f$ and $\rho$ be complex valued functions, analytic in the domain $\Phi=$ $J \times U$. Then the fractional coupled system (3.18) is equivalent to the transformed fractional coupled system (3.22) if and only if (3.23) holds.

Corollary 3.4. Let

$$
\begin{gathered}
a_{2}=0, \\
b_{2}=\frac{2 \rho_{1 z} a_{1} \rho_{2}-2 \rho_{2 z} a_{1} \rho_{1}}{\rho_{1}^{2}+\rho_{2}^{2}}, \\
c_{2}=\frac{\rho_{1 z z} a_{1} \rho_{2}+\rho_{2 z z} a_{1} \rho_{1}+\rho_{1 z}\left(b_{1} \rho_{2}-b_{2} \rho_{1}\right)-\rho_{2 z}\left(b_{1} \rho_{1}+b_{2} \rho_{2}\right)}{\rho_{1}^{2}+\rho_{2}^{2}} .
\end{gathered}
$$

Then the fractional coupled system (3.18) is equivalent to the transformed fractional uncoupled system

$$
\begin{gathered}
D^{\alpha} \bar{v}=\bar{a}_{1} \bar{v}_{z z}+\bar{b}_{1} \bar{v}_{z}+\bar{c}_{1} \bar{v}+\bar{f}_{1}, \\
D^{\alpha} \bar{w}=\bar{a}_{1} \bar{w}_{z z}+\bar{b}_{1} \bar{w}_{z}+\bar{c}_{1} \bar{w}+\bar{f}_{2},
\end{gathered}
$$

where $\bar{a}_{1}, \bar{b}_{1}$, and $\bar{c}_{1}$ are defined in (3.23).

\subsection{Nonequivalence Transforms}

Here, we shall show that the transform (3.14) reduces (2.6) into nonhomogeneous equation. And Hence we obtain nonhomogeneous system. Substituting (3.14) in (2.6), we have

$$
D^{\alpha} \bar{u}(t, z)=\bar{a}(t, z) \bar{u}_{z z}+\bar{b}(t, z) \bar{u}_{z}+\bar{c}(t, z) \bar{u}+g(t, z)
$$

where

$$
\bar{a}=a, \quad \bar{b}=b+2 a \frac{\rho_{z}}{\rho}, \quad \bar{c}=c+a \frac{\rho_{z z}}{\rho}+b \frac{\rho_{z}}{\rho}, \quad g(t, z)=\frac{\alpha \rho_{t}}{\rho} I^{1-\alpha} \bar{u}:=g_{1}(t, z)+i g_{2}(t, z) .
$$

A computation implies the following system:

$$
\begin{aligned}
& D^{\alpha} v=a_{1} v_{z z}-a_{2} w_{z z}+b_{1} v_{z}-b_{2} w_{z}+c_{1} v-c_{2} w+g_{1}, \\
& D^{\alpha} w=a_{1} w_{z z}+a_{2} v_{z z}+b_{1} w_{z}+b_{2} v_{z}+c_{1} w+c_{2} v+g_{2} .
\end{aligned}
$$

Now in view of (3.19)-(3.21), we have the system

$$
\begin{aligned}
& D^{\alpha} \bar{v}=\bar{a}_{1} \bar{v}_{z z}-\bar{a}_{2} \bar{w}_{z z}+\bar{b}_{1} \bar{v}_{z}-\bar{b}_{2} \bar{w}_{z}+\bar{c}_{1} \bar{v}-\bar{c}_{2} \bar{w}+\bar{g}_{1} \\
& D^{\alpha} \bar{w}=\bar{a}_{1} \bar{w}_{z z}+\bar{a}_{2} \bar{v}_{z z}+\bar{b}_{1} \bar{w}_{z}+\bar{b}_{2} \bar{v}_{z}+\bar{c}_{1} \bar{w}+\bar{c}_{2} \bar{v}+\bar{g}_{2}
\end{aligned}
$$


where

$$
\begin{gathered}
\bar{a}_{1}=a_{1}, \quad \bar{a}_{2}=a_{2}, \\
\bar{b}_{1}=b_{1}+\frac{2 \rho_{1 z}\left(a_{1} \rho_{1}+a_{2} \rho_{2}\right)+2 \rho_{2 z}\left(a_{1} \rho_{2}-a_{2} \rho_{1}\right)}{\rho_{1}^{2}+\rho_{2}^{2}}, \\
\bar{b}_{2}=b_{2}-\frac{2 \rho_{1 z}\left(a_{1} \rho_{2}-a_{2} \rho_{1}\right)+2 \rho_{2 z}\left(a_{2} \rho_{2}-a_{1} \rho_{1}\right)}{\rho_{1}^{2}+\rho_{2}^{2}}, \\
\bar{c}_{1}=c_{1}+\frac{\rho_{1 z z}\left(a_{1} \rho_{1}+a_{2} \rho_{2}\right)+\rho_{2 z z}\left(a_{1} \rho_{2}-a_{2} \rho_{1}\right)+\rho_{1 z}\left(b_{1} \rho_{1}+b_{2} \rho_{2}\right)+\rho_{2 z}\left(b_{1} \rho_{2}-b_{2} \rho_{1}\right)}{\rho_{1}^{2}+\rho_{2}^{2}}, \\
\bar{c}_{2}=c_{2}-\frac{\rho_{1 z z}\left(a_{1} \rho_{2}-a_{2} \rho_{1}\right)+\rho_{2 z z}\left(a_{1} \rho_{1}-a_{2} \rho_{2}\right)+\rho_{1 z}\left(b_{1} \rho_{2}-b_{2} \rho_{1}\right)-\rho_{2 z}\left(b_{1} \rho_{1}+b_{2} \rho_{2}\right)}{\rho_{1}^{2}+\rho_{2}^{2}}, \\
\bar{g}_{1}=\frac{\rho_{1}\left(g_{1}-h_{1}\right)+\rho_{2}\left(g_{2}-h_{2}\right)}{\rho_{1}^{2}+\rho_{2}^{2}}, \\
\bar{g}_{2}=\frac{\rho_{2}\left(g_{1}-h_{1}\right)+\rho_{1}\left(g_{2}-h_{2}\right)}{\rho_{1}^{2}+\rho_{2}^{2}} .
\end{gathered}
$$

Thus we receive the following result.

Theorem 3.5. Let $a \neq 0, b, c, u$, and $\rho$ be complex valued functions, analytic in the domain $\Phi=J \times U$. Then the fractional coupled system (3.28) is equivalent to the transformed fractional coupled system (3.29) if and only if (3.30) holds.

Corollary 3.6. Let

$$
\begin{gathered}
a_{2}=0, \\
b_{2}=\frac{2 \rho_{1 z} a_{1} \rho_{2}-2 \rho_{2 z} a_{1} \rho_{1}}{\rho_{1}^{2}+\rho_{2}^{2}}, \\
c_{2}=\frac{\rho_{1 z z} a_{1} \rho_{2}+\rho_{2 z z} a_{1} \rho_{1}+\rho_{1 z}\left(b_{1} \rho_{2}-b_{2} \rho_{1}\right)-\rho_{2 z}\left(b_{1} \rho_{1}+b_{2} \rho_{2}\right)}{\rho_{1}^{2}+\rho_{2}^{2}} .
\end{gathered}
$$

Then the fractional coupled system (3.28) is equivalent to the transformed fractional uncoupled system

$$
\begin{gathered}
D^{\alpha} \bar{v}=\bar{a}_{1} \bar{v}_{z z}+\bar{b}_{1} \bar{v}_{z}+\bar{c}_{1} \bar{v}+\bar{g}_{1}, \\
D^{\alpha} \bar{w}=\bar{a}_{1} \bar{w}_{z z}+\bar{b}_{1} \bar{w}_{z}+\bar{c}_{1} \bar{w}+\bar{g}_{2},
\end{gathered}
$$

where $\bar{a}_{1}, \bar{b}_{1}$, and $\bar{c}_{1}$ are defined in (3.30). 


\section{Existence and Uniqueness}

In this section we shall discuss the existence and uniqueness of solution for the linear and nonlinear systems. For this purpose, we need the following concept.

Definition 4.1 (see [38]). A vector-valued function $X(t, x)$ is said to satisfy a Lipschitz condition in a region $R$ in $(t, x)$-space if, for some constant $L$ (called the Lipschitz constant), we have

$$
\|X(t, x)-X(t, y)\| \leq L\|x-y\|
$$

whenever $(t, x),(t, y) \in \mathcal{R}$.

A convex domain $D$ means that for any two points $x$ and $y$ in $D,(1-t) x+t y \in D$ for $0 \leq t \leq 1$.

Lemma 4.2 (see [38]). If $X(t, x)$ has continuous partial derivatives on a bounded closed convex domain $D$, then it satisfies a Lipschitz condition.

Theorem 4.3. Assume that all the functions in the system (3.9) are analytic in the domain $\Phi$. Then the system (3.9)-(3.10) has a unique solution in $\boldsymbol{\Phi}$.

Proof. System (3.9) can be assumed as in a matrix form

$$
\left(\begin{array}{c}
D^{\alpha} \bar{v} \\
D^{\alpha} \bar{w}
\end{array}\right)=\left(\begin{array}{cccccc}
\bar{a}_{1} & -\bar{a}_{2} & \bar{b}_{1} & -\bar{b}_{2} & \bar{c}_{1} & -\bar{c}_{2} \\
\bar{a}_{2} & \bar{a}_{1} & \bar{b}_{2} & \bar{b}_{1} & \bar{c}_{2} & \bar{c}_{1}
\end{array}\right)\left(\begin{array}{c}
\bar{v}_{z z} \\
\bar{w}_{z z} \\
\bar{v}_{z} \\
\bar{w}_{z} \\
\bar{v} \\
\bar{w}
\end{array}\right)
$$

$$
:=A Z \text {, }
$$

where $Z=\left(Z_{1}(t, z), \ldots, Z_{6}(t, z)\right)^{T}$. Since the domain $\Phi=J \times U$ is bounded closed convex and all the functions $Z_{i}, i=1, \ldots, 6$ are analytic in $\Phi$ then in view of Lemma $4.2, Z$ satisfies a Lipschitz condition. Hence by the Banach fixed point theorem, (4.2) has a unique solution and has a unique solution as well as system (3.9). This completes the proof.

Theorem 4.4. Let the functions in the system (3.22) be analytic in the domain $\boldsymbol{\Phi}$. Moreover let $\bar{f}$ be a Lipschitz function. Then the system (3.22)-(3.23) has a unique solution in $\boldsymbol{\Phi}$. 
Proof. System (3.22) can be written in a matrix form

$$
\begin{aligned}
\left(\begin{array}{c}
D^{\alpha} \bar{v} \\
D^{\alpha} \bar{w}
\end{array}\right) & =\left(\begin{array}{cccccc}
\bar{a}_{1} & -\bar{a}_{2} & \bar{b}_{1} & -\bar{b}_{2} & \bar{c}_{1} & -\bar{c}_{2} \\
\bar{a}_{2} & \bar{a}_{1} & \bar{b}_{2} & \bar{b}_{1} & \bar{c}_{2} & \bar{c}_{1}
\end{array}\right)\left(\begin{array}{c}
\bar{v}_{z z} \\
\bar{w}_{z z} \\
\bar{v}_{z} \\
\bar{w}_{z} \\
\bar{v} \\
\bar{w}
\end{array}\right)+\left(\begin{array}{c}
\bar{f}_{1} \\
\bar{f}_{2}
\end{array}\right) \\
& :=A Z+F .
\end{aligned}
$$

Hence Lemma 4.2 and Banach fixed point theorem imply the uniqueness of (4.3) and consequently (3.22). This completes the proof.

\section{Applications}

In this section, we shall illustrate some examples.

Example 5.1. Consider the equation

$$
D^{\alpha} u(t, z)=A z^{2} u_{z z}+B z u_{z}+C u,
$$

where $A \neq 0, B$ and $C$ are real constants, and the complex transform

$$
u(t, z)=z^{2} \bar{u}(t, z):=\left(\sigma_{1}+i \sigma_{2}\right)(\bar{v}+i \bar{w}) .
$$

Equation (5.1) reduces to the homogeneous type

$$
D^{\alpha} \bar{u}(t, z)=\bar{A} z^{2} u_{z z}+\bar{B} z u_{z}+\bar{C} u,
$$

where

$$
\bar{A}=A, \quad \bar{B}=(4 A+B), \quad \bar{C}=2 A+2 B+C .
$$

Hence (5.3) can be translated to the uncoupled system

$$
\begin{gathered}
D^{\alpha} \bar{v}=\bar{A} \sigma_{1} \bar{v}_{z z}+\bar{B}\left( \pm \sqrt{\frac{r+\sigma_{1}}{2}}\right) \bar{v}_{z}+\bar{C} \bar{v} \\
D^{\alpha} \bar{w}=\bar{A} \sigma_{2} \bar{w}_{z z}+\bar{B}\left( \pm \sqrt{\frac{r-\sigma_{1}}{2}}\right) \bar{w}_{z}+\bar{C} \bar{w}
\end{gathered}
$$


where $r=\sqrt{\sigma_{1}^{2}+\sigma_{2}^{2}}$. Furthermore, it can be represented as coupled system

$$
\begin{aligned}
& D^{\alpha} \bar{v}=\bar{A} \sigma_{1} \bar{v}_{z z}-\bar{A} \sigma_{2} \bar{w}_{z z}+\bar{B}\left( \pm \sqrt{\frac{r+\sigma_{1}}{2}}\right) v_{z}-\bar{B}\left( \pm \sqrt{\frac{r-\sigma_{1}}{2}}\right) \bar{w}_{z}+\bar{C} \bar{v}-\bar{C} \bar{w} \\
& D^{\alpha} \bar{w}=\bar{A} \sigma_{1} \bar{w}_{z z}+\bar{A} \sigma_{2} \bar{v}_{z z}+\bar{B}\left( \pm \sqrt{\frac{r+\sigma_{1}}{2}}\right) \bar{w}_{z}+\bar{B}\left( \pm \sqrt{\frac{r-\sigma_{1}}{2}}\right) \bar{v}_{z}+\bar{C} \bar{w}+\bar{C} \bar{v}
\end{aligned}
$$

Example 5.2. Consider the equation

$$
D^{\alpha} u(t, z)=A t z^{2} u_{z z}+B t z u_{z}+C t u+e^{i t}, \quad t \in[a, 1], a \neq 0,
$$

where $A \neq 0, B$ and $C$ are real constants, and the complex transform

$$
u(t, z)=t z^{2} \bar{u}(t, z):=t\left(\rho_{1}+i \rho_{2}\right)(\bar{v}+i \bar{w}) .
$$

Thus (5.7) becomes

$$
D^{\alpha} \bar{u}(t, z)=\bar{A} t z^{2} u_{z z}+\bar{B} t z u_{z}+\bar{C} t u+\bar{f}
$$

where

$$
\begin{gathered}
\bar{A}=A, \quad \bar{B}=(4 A+B), \quad \bar{C}=2 A+2 B+C, \\
\bar{f}=e^{i t}-\frac{\alpha I^{1-\alpha} \bar{u}}{t}:=\left(\cos t-\phi_{1}\right)+i\left(\sin t-\phi_{2}\right):=\bar{f}_{1}+i \bar{f}_{2} .
\end{gathered}
$$

Hence (5.7) can be formulated to the uncoupled system

$$
\begin{aligned}
D^{\alpha} \bar{v} & =\bar{A} \rho_{1} \bar{v}_{z z}+\bar{B} t\left( \pm \sqrt{\frac{r+\rho_{1}}{2}}\right) \bar{v}_{z}+\bar{C} t \bar{v}+\bar{f}_{1} \\
D^{\alpha} \bar{w} & =\bar{A} \rho_{2} \bar{w}_{z z}+\bar{B} t\left( \pm \sqrt{\frac{r-\rho_{1}}{2}}\right) \bar{w}_{z}+\bar{C} t \bar{w}+\bar{f}_{2}
\end{aligned}
$$

where $r=\sqrt{\rho_{1}^{2}+\rho_{2}^{2}}$. Furthermore, it can be represented as coupled system

$$
\begin{aligned}
& D^{\alpha} \bar{v}=\bar{A} \rho_{1} \bar{v}_{z z}-\bar{A} \rho_{2} \bar{w}_{z z}+\bar{B} t\left( \pm \sqrt{\frac{r-\rho_{1}}{2}}\right) v_{z}-\bar{B} t\left( \pm \sqrt{\frac{r-\rho_{1}}{2}}\right) \bar{w}_{z}+\bar{C} t \bar{v}-\bar{C} t \bar{w}+\bar{f}_{1}, \\
& D^{\alpha} \bar{w}=\bar{A} \rho_{1} \bar{w}_{z z}+\bar{A} \rho_{2} \bar{v}_{z z}+\bar{B} t\left( \pm \sqrt{\frac{r+\rho_{1}}{2}}\right) \bar{w}_{z}+\bar{B} t\left( \pm \sqrt{\frac{r-\rho_{1}}{2}}\right) \bar{v}_{z}+\bar{C} t \bar{w}+\bar{C} t \bar{v}+\bar{f}_{2} .
\end{aligned}
$$


Next example is a time fractional diffusion equation, which describes the reconstruction of a source. Recently, for real case, this equation is studied by Wei and Zhang [39].

Example 5.3. Consider the fractional diffusion equation

$$
D^{\alpha} u(t, z)=u_{z z}+f(z) p(t)
$$

where $u(t, z)$ is the solute concentration, $p(t)$ is the source term, and $t \in(0, T), z \in U$. Equation (5.13) is a special case of (3.13) with $a(t, z)=1, b(t, z)=c(t, z)=0$; thus it can be reduced to the coupled system

$$
\begin{aligned}
D^{\alpha} \bar{v} & =\bar{v}_{z z}-\bar{b}_{1} \bar{v}_{z}-\bar{b}_{2} \bar{w}_{z}+\bar{c}_{1} \bar{v}-\bar{c}_{2} \bar{w}+\bar{f}_{1}, \\
D^{\alpha} \bar{w} & =\bar{w}_{z z}+\bar{b}_{1} \bar{w}_{z}+\bar{b}_{2} \bar{v}_{z}+\bar{c}_{1} \bar{w}+\bar{c}_{2} \bar{v}+\bar{f}_{2},
\end{aligned}
$$

where $\bar{f}=\left(f_{p}(t) / \rho\right)-\left(\alpha \rho_{t} / \rho\right) I^{1-\alpha} \bar{u}:=\bar{f}_{1}+i \bar{f}_{2^{\prime}}$

$$
\begin{gathered}
\bar{a}_{1=1}, \quad \bar{a}_{2}=0, \quad \bar{b}_{1}=\frac{2 \rho_{1 z}\left(a_{1} \rho_{1}\right)+2 \rho_{2 z}\left(a_{1} \rho_{2}\right)}{\rho_{1}^{2}+\rho_{2}^{2}}, \\
\bar{b}_{2}=-\frac{2 \rho_{1 z}\left(a_{1} \rho_{2}\right)+2 \rho_{2 z}\left(-a_{1} \rho_{1}\right)}{\rho_{1}^{2}+\rho_{2}^{2}}, \quad \bar{c}_{1}=\frac{\rho_{1 z z}\left(a_{1} \rho_{1}\right)+\rho_{2 z z}\left(a_{1} \rho_{2}\right)}{\rho_{1}^{2}+\rho_{2}^{2}}, \\
\bar{c}_{2}=-\frac{\rho_{1 z z}\left(a_{1} \rho_{2}\right)+\rho_{2 z z}\left(a_{1} \rho_{1}\right)}{\rho_{1}^{2}+\rho_{2}^{2}}, \quad \bar{f}_{1}=\frac{\rho_{1}\left(f_{1}-h_{1}\right)+\rho_{2}\left(f_{2}-h_{2}\right)}{\rho_{1}^{2}+\rho_{2}^{2}}, \\
\bar{f}_{2}=\frac{\rho_{2}\left(f_{1}-h_{1}\right)+\rho_{1}\left(f_{2}-h_{2}\right)}{\rho_{1}^{2}+\rho_{2}^{2}} .
\end{gathered}
$$

\section{Conclusion}

We suggested two types of complex transforms for systems of fractional differential equations. We concluded that the complex fractional differential equations can be transformed into coupled and uncoupled system of homogeneous and nonhomogeneous types. Moreover, the existence and uniqueness of these systems are studied by using the Banach fixed point theorem. Some examples are illustrated.

\section{Acknowledgments}

The author is thankful to the referees for helpful suggestions for the improvement of this paper.

\section{References}

[1] M. Cassol, S. Wortmann, and U. Rizza, "Analytic modeling of two-dimensional transient atmospheric pollutant dispersion by double GITT and Laplace transform techniques," Environmental Modelling $\mathcal{E}$ Software, vol. 24, no. 1, pp. 144-151, 2009. 
[2] C. Delong, "A novel fingerprint encryption algorithm based on chaotic system and fractional Fourier transform," International Conference on Machine Vision and Human-machine Interface, pp. 186-171, 2010.

[3] A. Bekir, "New exact travelling wave solutions of some complex nonlinear equations," Communications in Nonlinear Science and Numerical Simulation, vol. 14, no. 4, pp. 1069-1077, 2009.

[4] P. R. Gordoa, A. Pickering, and Z. N. Zhu, "Bäcklund transformations for a matrix second Painlevé equation," Physics Letters A, vol. 374, no. 34, pp. 3422-3424, 2010.

[5] S. Sivasubramanian, M. Darus, and R. W. Ibrahim, "On the starlikeness of certain class of analytic functions," Mathematical and Computer Modelling, vol. 54, no. 1-2, pp. 112-118, 2011.

[6] H. M. Srivastava, M. Darus, and R. W. Ibrahim, "Classes of analytic functions with fractional powers defined by means of a certain linear operator," Integral Transforms and Special Functions, vol. 22, no. 1, pp. 17-28, 2011.

[7] Z. Li and J. He, "Application of the fractional complex transform to fractional differential equations," Nonlinear Science Letters A, vol. 2, no. 3, pp. 121-126, 2011.

[8] R. Y. Molliq and B. Batiha, "Approximate analytic solutions of fractional Zakharov-Kuznetsov equations by fractional complex transform," International Journal of Engineering and Technology, vol. 1, no. 1, pp. 1-13, 2012.

[9] W. G. Glöckle and T. F. Nonnenmacher, "Fox function representation of non-Debye relaxation processes," Journal of Statistical Physics, vol. 71, no. 3-4, pp. 741-757, 1993.

[10] R. W. Ibrahim, "Existence and uniqueness of holomorphic solutions for fractional Cauchy problem," Journal of Mathematical Analysis and Applications, vol. 380, no. 1, pp. 232-240, 2011.

[11] R. W. Ibrahim, “On holomorphic solution for space- and time-fractional telegraph equations in complex domain," Journal of Function Spaces and Applications, vol. 2012, Article ID 703681, 10 pages, 2012.

[12] R. W. Ibrahim, "Approximate solutions for fractional differential equation in the unit disk," Electronic Journal of Qualitative Theory of Differential Equations, vol. 64, pp. 1-11, 2011.

[13] S. Momani and Z. Odibat, "Comparison between the homotopy perturbation method and the variational iteration method for linear fractional partial differential equations," Computers $\mathcal{E}$ Mathematics with Applications, vol. 54, no. 7-8, pp. 910-919, 2007.

[14] R. Y. Molliq, M. S. M. Noorani, and I. Hashim, "Variational iteration method for fractional heat- and wave-like equations," Nonlinear Analysis. Real World Applications, vol. 10, no. 3, pp. 1854-1869, 2009.

[15] K. Sayevand, A. Golbabai, and A. Yildirim, "Analysis of differetial equations of fractional order," Applied Mathematical Modelling, vol. 36, pp. 4356-4364, 2012.

[16] H. Jafari and M. A. Firoozjaee, "Homotopy analysis method for solving KdV equations," Surveys in Mathematics and its Applications, vol. 5, pp. 89-98, 2010.

[17] R. Metzler, E. Barkai, and J. Klafter, "Deriving fractional Fokker-Planck equations from a generalised master equation," Europhysics Letters, vol. 46, no. 4, pp. 431-436, 1999.

[18] E. K. Lenzi, L. R. Evangelista, and G. Barbero, "Fractional diffusion equation and impedance spectroscopy of electrolytic cells," The Journal of Physical Chemistry B, vol. 113, no. 33, pp. 11371-11374, 2009.

[19] J. R. Macdonald, L. R. Evangelista, E. K. Lenzi, and G. Barbero, “Comparison of impedance spectroscopy expressions and responses of alternate anomalous poisson-nernst-planck diffusion equations for finite-length situations," The Journal of Physical Chemistry C, vol. 115, no. 15, pp. 7648-7655, 2011.

[20] P. A. Santoro, J. L. de Paula, E. K. Lenzi, and L. R. Evangelista, "Anomalous diffusion governed by a fractional diffusion equation and the electrical response of an electrolytic cell," Journal of Chemical Physics, vol. 135, Article ID 114704, 5 pages, 2011.

[21] J. T. Machado, V. Kiryakova, and F. Mainardi, "Recent history of fractional calculus," Communications in Nonlinear Science and Numerical Simulation, vol. 16, no. 3, pp. 1140-1153, 2011.

[22] R. Hilfer, R. Metzler, A. Blumen, and J. Klafter, "Preface," Chemical Physics, vol. 284, no. 1-2, pp. 1-2, 2002.

[23] J. Klafter, S. C. Lim, and R. Metzler, Eds., Fractional Dynamics: Recent Advances, World Scientific, Publishing Company, Singapore, 2011.

[24] R. Metzler and J. Klafter, "From a generalized Chapman-Kolmogorov equation to the fractional KleinKramers equation," The Journal of Physical Chemistry B, vol. 104, pp. 3851-3857, 2000.

[25] I. Podlubny, Fractional Differential Equations, Academic Press, London, UK, 1999.

[26] A. A. Kilbas, H. M. Srivastava, and J. J. Trujillo, Theory and Applications of Fractional Differential Equations, Elsevier, Amsterdam, The Netherlands, 2006. 
[27] H. M. Srivastava and S. Owa, Univalent Functions, Fractional Calculus, and Their Applications, Halsted Press, John Wiley \& Sons, New York, NY, USA, 1989.

[28] K. Diethelm, The Analysis of Fractional Differential Equations, Springer, Berlin, Germany, 2010.

[29] A. N. Kochuber,, "The Cauchy problem for evolution equations of fractional order," Differential Equations, vol. 25, pp. 967-974, 1989.

[30] A. N. Kochubel̆, “Diffusion of fractional order," Differential Equations, vol. 26, no. 4, pp. 485-492, 1990.

[31] R. Metzler and J. Klafter, "The random walk's guide to anomalous diffusion: a fractional dynamics approach," Physics Reports, vol. 339, no. 1, pp. 1-77, 2000.

[32] G. M. Zaslavsky, "Fractional kinetic equation for Hamiltonian chaos," Physica D, vol. 76, no. 1-3, pp. 110-122, 1994.

[33] F. Mainardi, G. Pagnini, and R. Gorenflo, "Some aspects of fractional diffusion equations of single and distributed order," Applied Mathematics and Computation, vol. 187, no. 1, pp. 295-305, 2007.

[34] F. Mainardi, A. Mura, G. Pagnini, and R. Gorenflo, Sub-diffusion equations of fractional order and their fundamental solutions, Invited lecture by F. Mainardi at the 373. WEHeraeus- Seminar on Anomalous Transport: Experimental Results and Theoretical Challenges, Physikzentrum Bad-Honnef (Germany), July 2006.

[35] T. Sandev, R. Metzler, and Z. Tomovski, "Fractional diffusion equation with a generalized RiemannLiouville time fractional derivative," Journal of Physics A, vol. 44, no. 25, pp. 1-21, 2011.

[36] R. W. Ibrahim, "Solutions of fractional diffusion problems," Electronic Journal of Differential Equations, vol. 147, pp. 1-11, 2010.

[37] M. Merdan, "Solutions of time-fractional reaction-diffusion equation with modified RiemannLiouville derivative," International Journal of Physical Sciences, vol. 7, pp. 2317-2326, 2012.

[38] J. Hu and W.-P. Li, Theory of Ordinary Differential Equations, The Hong Kong University of Science and Technology, 2005.

[39] T. Wei and Z. Zhang, "Reconstruction of a time-dependent so urce term in a time-fractional diffusion equation," Engineering Analysis with Boundary Elements, vol. 37, no. 1, pp. 23-31, 2013. 


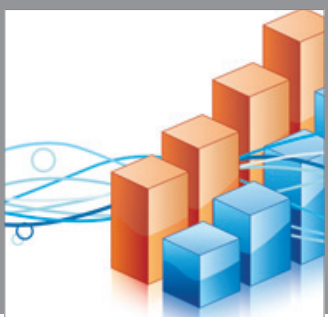

Advances in

Operations Research

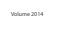

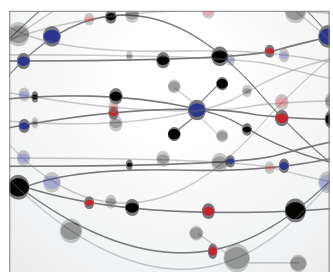

\section{The Scientific} World Journal
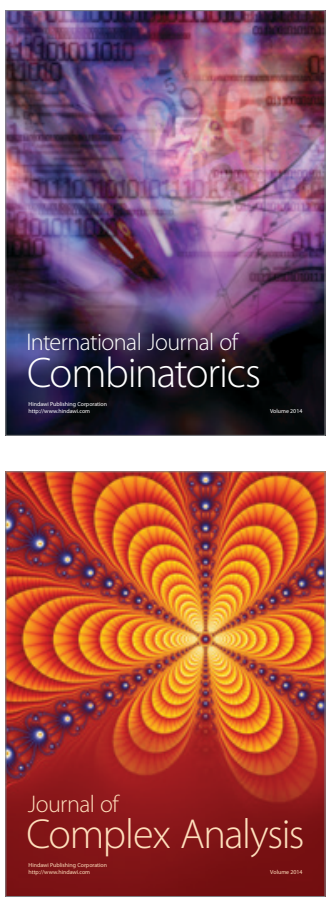

International Journal of

Mathematics and

Mathematical

Sciences
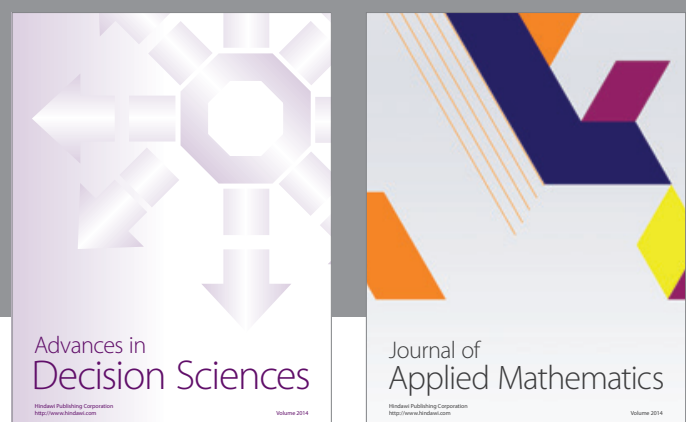

Journal of

Applied Mathematics
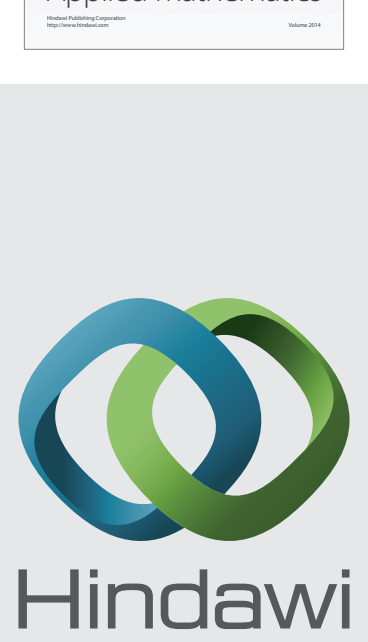

Submit your manuscripts at http://www.hindawi.com
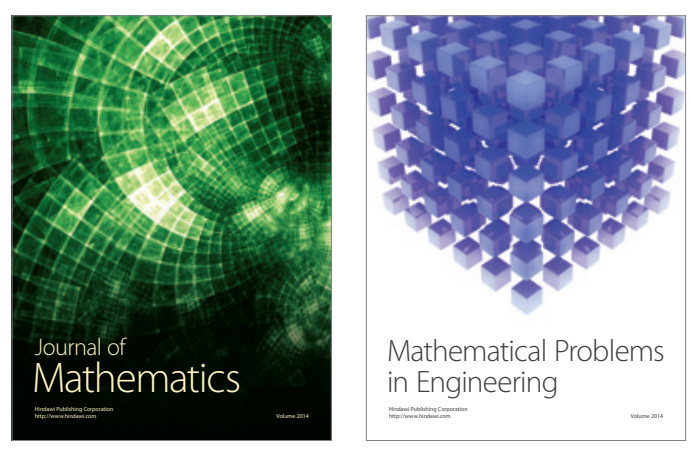

Mathematical Problems in Engineering
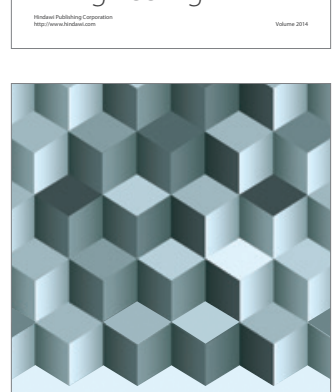

Journal of

Function Spaces
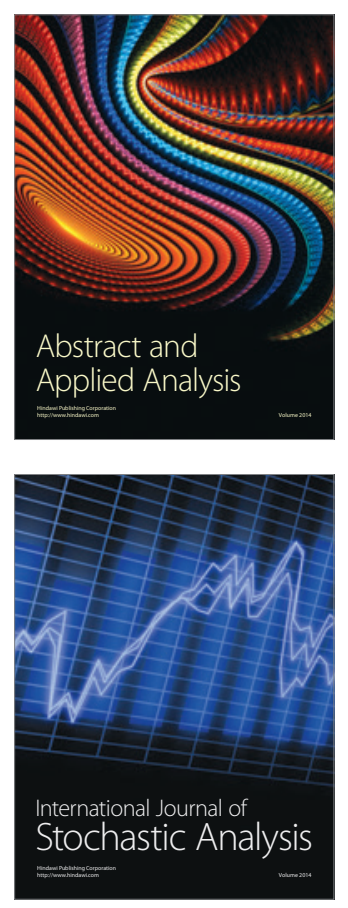

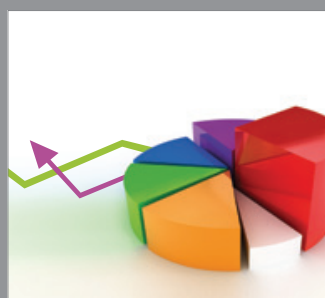

ournal of

Probability and Statistics

Promensencen
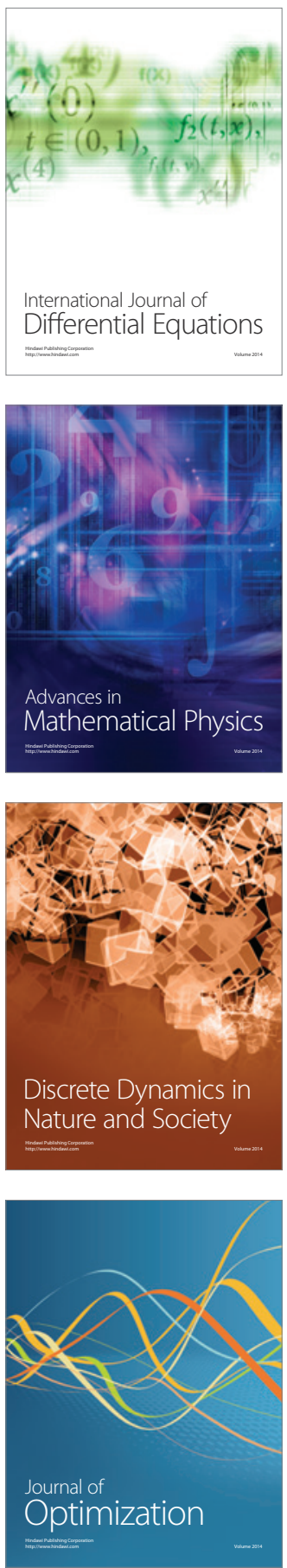